\title{
Artificial Intelligence Applications and Future Research Directions
}

\author{
K .G. Rani Roopha Devi' R.Mahendra Chozhan' R. Murugesan
}

\begin{abstract}
This is an extensive study of Artificial Intelligence applications. It offers artificial neural networks (ANN) taxonomy and supplies investigators with current knowledge and raising needs in ANN based research applications and concentration for investigators. In addition, this study offers an ANN application contributions, challenges, performance comparison and evaluation. This study is demonstrated various ANN applications in diverse disciplines comprise science, computing, medicine, environmental, engineering, climate, technology, mining, arts, nanotechnology, business and so on. Based on this review, it is identified that neural network models like Feedback propagation and Feed forward artificial neural networks performs effectually in human problems based application. Henceforth, feed forward and feed backward propagation ANN focuses on research sourced on data analysis parameters such as accuracy, fault tolerance, latency, volume, convergence, scalability and performance. However, this study suggests that indeed of utilizing single method, future investigation concentrates on merging ANN models into cloud and dentistry based network wide application.
\end{abstract}

Keywords- Artificial intelligence; Artificial Neural networks; feed forward; feed backward; ANN applications; cloud fusion; Dentistry.

\section{INTRODUCTION}

In current times, ANN has turned as a most accepted and essential model for clustering, classification, prediction and pattern detection in diverse applications. ANN are one kind of machine learning model and becomes most competitive model to traditional statistical and regression models based on its utility [1]. At present, AI (deep learning, NN, machine learning, robotic), big data, internet, information security, forensic science and cloud computing are hotspots and prevailing topics on information and communication technology.

In general, ANN can be measured based on data analysis parameters such as speed, accuracy, performance, latency, volume, tolerance, convergence and scalability. The superior ANN potential is high speed processing is given in enormous parallel execution and this is enlarged based on the research need in this field. ANNs is modelled for natural language processing, image recognition and so on.

Revised Manuscript Received on July 08, 2019

K .G. Rani Roopha Devi, Research Scholar, Madurai Kamaraj University, Madurai.India

Dr.R.Mahendra Chozhan ${ }^{2}$ Chozhan Dental Clinic, Kodaikanal, Periyakulam, Lakshmipuram, India

Dr. R. Murugesan Associate Professor, Department of Computer Science, C.P.A College, Bodinayakanur, India.
In recent times, ANNs are extensively utilized for universal function approximation in numerical paradigms as the essential properties of adaptability, self-learning, non-linearity, fault tolerance and input development in mapping.

Data analysis parameters offer reason why it is effective and successful in offering high capability in dealing noncomplex and complex crisis in numerous aspects. It is competent of dealing crisis that is encountered in science, education, agriculture, medical science, management, finance, engineering, commodity and art. It as well includes problems in transportation, manufacturing, banking, computer security, management, marketing, insurance, energy and challenges are not be solved using computational capability of conventional mathematics procedures [2].

Indeed of wide ANN applications, there is a rising necessitate to resolve the problem of accepting systematic in ANNs phase to enhance performance. For example, a method to resolve major crisis and data sets selection (volume, large or otherwise), accuracy, standardization, instrument, inputs type, division, and pre-processing, processing, validations and output approaches. Evolution of AI is shown in fig 1.

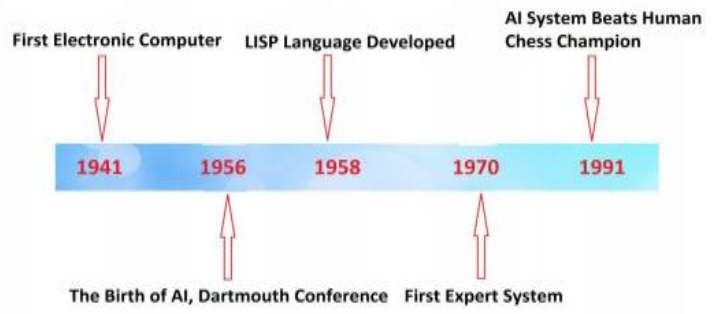

Fig 1: Evolution of AI

As well, other primary challenges which are common while modelling of ANN have received attention and need more studies in future. Techniques can progress robust model designing, enhancing pattern transparency and facilitating functional knowledge from ANNs. Challenges of enhancing extrapolation ability are design of novel approaches to uncertainty and enhancing convergences.

- Progressing robust model design: model robustness determines predictive ANN capability kinds in simplifying data range utilized for training.

\section{- Enhancing model transparency and facilitating knowledge extraction using}


trained ANNs: it specifies interpreting ANN models possibility that offers deep knowledge of modelling inputs to outputs.

- Enhancing extrapolation ability: ANN model extrapolation is the models ability to forecast precisely outward data range utilized for ANN model calibration.

- New methodologies to uncertainty: certain ANN limitations comprise predictions uncertainty which is not considered. While uncertainty is not accounted, it turns to be more complex to measure predictions quality of ANN, which can significantly, restricts or diminishes its efficacy.

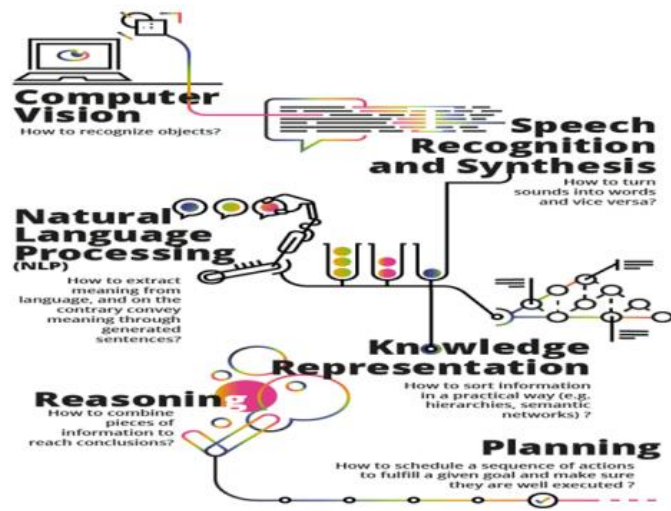

Fig 2: AI abilities

\section{ARTIFICIAL NEURAL NETWORKS}

In recent times, ANN application has turns to be more popular in diverse fields of human necessities. Numerous organizations are examining NNs to resolve crisis in diverse fields and economic sector which conventionally comes under operations research [3]. Artificial intelligence applications make it more unique for analyses by academics in arts field distinct from science and engineering. For instance, in current times, AI has been widely utilized to optimization crisis in various fields such as petroleum exploration, industrial production and business setting as in fig 2 .

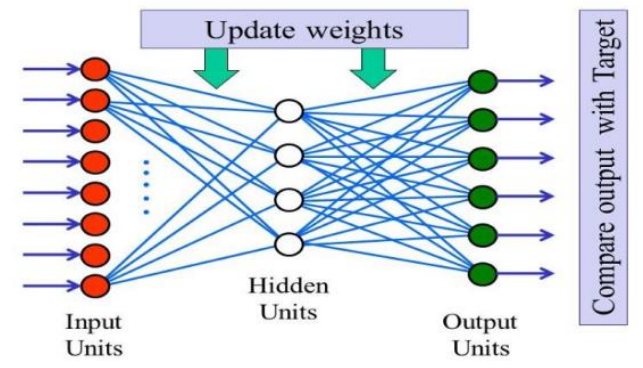

Fig 3: Artificial neural network representation

A superior benefit of ANN application is, it makes model to be used easily and extremely accurate from complex systems with huge inputs. ANN is considered as an extremely helpful model for problem-solving and ML. ANN is similar to biological nervous systems of human brain as in fig 3 . Research interest towards brain functionality has increased worldwide. ANN is comparable machine generated to function in similar way as that of human brain which provides task interest.

For instance, "brain is highly efficient and big. Brain is information-processing which possess complex signal computing operations which are synchronized to carry out task [4]. Major component is information processing capability. It comprises numerous interconnected "neurons" that works to resolve certain crisis. Distinctive instance of $\mathrm{NN}$ human brain is associated for sending and receiving signals.

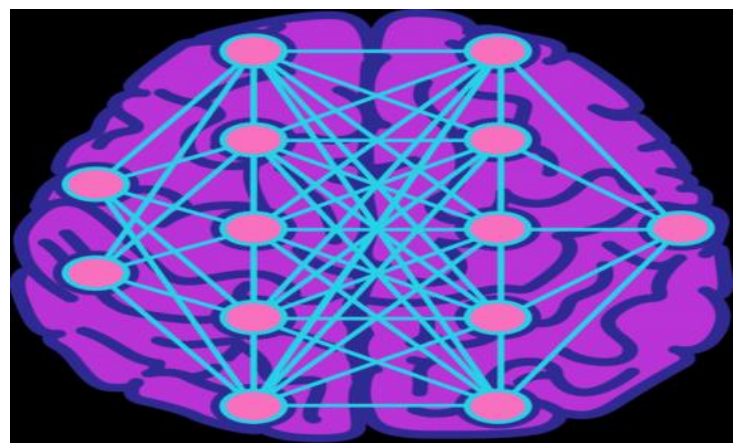

Fig 4: Brainstorming ANN representation

Brainstorming recognizes this scenario (web search, speech (unknown person and known person) such as human brain, image recognizing (object) such as language (translate) such as brain and carry out things such as bicycle, eating as in fig 4. To provide specific instance; it posses extensive use in some domains, like; hepatitis analysis data recovery; speech; faulty software telecommunications; multi-language messages interpretation; texture analysis; 3D object identification; mining; facial recognition; hand-written recognition. Therefore, it is understood from example [5] Specifically, it is modelled for some application like pattern recognition through learning procedure. It needs adjust synaptic association between neurons, similar to ANN. It works such as brain imitation.

Layers of NN are independent of one another; some layer possesses an arbitrary nodes. Amount of nodes is termed as bias node [6]. It is always fixed as equal to one. Bias node major functionality is to offer node with constant value which can be trainable, as well to inputs attained from network node. It facilitates activation function to move for training ANN successfully. NN is utilized as classifier; input/output nodes match's input/output classes. Moreover, when NN is utilized as function approximation, it has an input/output node. As well, number of hidden nodes necessarily higher than input.

\section{NEURAL NETWORKS CLASSIFICATION}

With the provided Neural networks (NNs) description, functionality and applications/ utilization, NNs have extensively applied to education, economics and numerous life factors. NNs are applicable in optimization approaches, data 
classification and intrusion detection. It is complex optimization confront. Investigators utilized ML in resolving classification crisis. NNs are outstanding identifier, they are suitable for predicting requirements including items list.

\section{A. Classification}

ANN is classified as Feed forward (FF) which is ML algorithm is organized which is alike of processing units. Every unit is associated to other units. These layers are connections with units are not all equal as every connection possesses diverse weight [7]. The network connections weight measures network knowledge. As well, units are termed as nodes. Information processing comprises of entry from input and moves via network, which flows from one layer to another till it moves to output. When NN functions usually it is operated as classifier, and there is no feedback among layers. Information is transmitted in one direction, i.e. from input to hidden nodes, and to output. These characteristics are called as feed forward neural networks.

\section{B. Deep learning}

AI has been in survival over numerous decades, and it is more extensive. AI comprises of ML and DL. ML is AI's subset; however, DL comes into existence which is ML's subset. In more specific, DL is AI based factors; DL is specified as ANN with composite multi-layers. Differentiation among DL and NNs like FFNNs and FBNNs is based on its characteristic. Deep learning is complex approach for connecting layers; as well possess enormous neurons count than networks to state difficult models, with computing power to train and with automatic feature extraction.

Henceforth, DL is defined as NN with extensive variables and essential network architecture of unsupervised convolutional NNs, pre-trained networks, recursive and recurrent NNs.

One amongst common NNs is CNN. It is standard NN that enhances over space through shared weights. It is modelled to distinguish images using convolutions which are considered as an object image. CNN holds multiple layers; comprising of pooling, fully-connected, convolutional and non-linearity layers. Last two layers have factors; nevertheless pooling and non-linearity layers do not possess parameters. Investigations determined an outstanding performance in ML. Image data application possesses broad image classification dataset, computer vision and language processing.

ANNs has important benefits over statistical methods, while comparing both of them relatively. Here, there exist no assumptions regarding data distribution and data properties. Henceforth, it is extensively useful in application [8]. As well, statistical models needs testing hypothesis, it do not necessitate hypothesis. There are reduction models, extensively flexible, nonlinear regression and discriminant models. Distinct of extreme learning machine, SVM and RF,
ANNs are fault tolerant. It deal with noise; it resolve non-linear crisis, as well, trained ANNs, generalized at higher speed and predictions. In addition, ANNs are scalable relative to extreme learning machine, SVM and RF.

\section{Modelling}

In general, modelling is process of presenting event or object as mathematical expression. Moreover, NN modelling is process of specifying the way of nervous system functions. $\mathrm{NN}$ is representation of how brain executes data [9]. It operates by simulating numerous processing units that look like various concepts of neurons version. It is an optimization hypothesis utilized in optimizing NN. In recent times, gradient descent utilized in updating weights, that is, modifying parameters to reduce Loss function.

So as to provide prediction model to identify expected outcome, loss function is essential, i.e. function class that reduced is termed as "loss functions". Appropriate method cast off for recognizing minimal function point specified as gradient descent. DNNs are recently utilized classifiers.

\section{General Applications}

Numerous ANN approaches are utilized in industries/academia to resolve challenges in speech \& pattern recognitions, computer vision, and detection and face alignment as in fig 5. These include;

\section{Speech recognition}

ANNs application has turn out to be divergence and unspoken in successes of communication recognition. ML algorithms are utilized in ASR (automatic speech recognition) and acoustic modelling.

\section{E. Computer vision}

It attempts providing computers to precisely carry out visual data resourcefully such as images. Foremost objective is to offer computers with brain functionality. Hypothetically, it refers to control which investigates data from images in ANNs. Computer vision based Sub domain comprises of object recognition and detection, position, estimation, scene reconstruction, event detection, image editing, restoration and statistical learning. Therefore, ANN is extremely useful [10].

\section{F. Pattern recognition}

Current enhancement in DL has provided ways to handle issues of PR. PR is field that concentrates in recognition of input sequence. PR is common idea that deals with diverse sub domains like regression, speech tagging, labelling and classification. There exists quickly rising requires for processing information and output. 


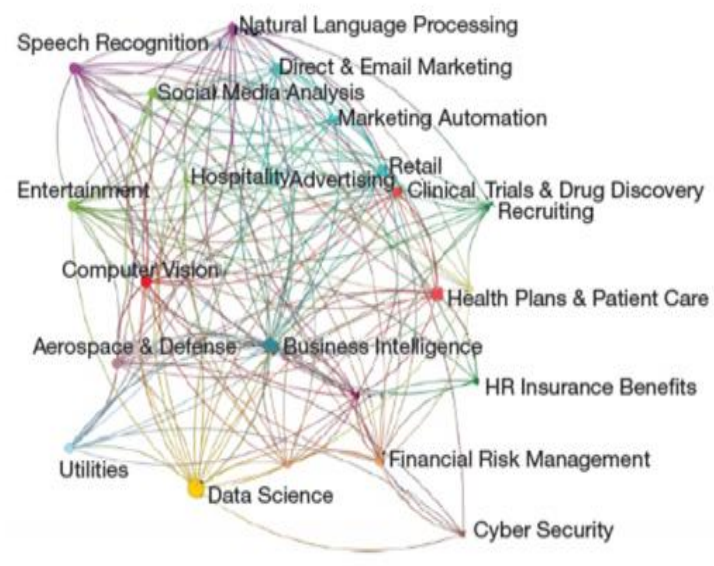

Fig 5: Applications of AI

\section{G. Face alignment}

Face alignment acts as an eminent role in visual applications. ANNs declared more success rate in face recognition, face alignment and other models are successes. DL interestingly utilized to describe genetic variants to recognize pathogenic variants. In general, merged annotation dependent depletion algorithm is generally utilized to understand coding and non-coding variants.

\section{H. Detection}

Detection in security, financial irregularity, medical diagnosis, objects, fault are improved via ANNs [11]. Therefore, it acts as a vital function in detection, specifically when utilized to breast cancer. ANN performance is moderately compared with approaches in crime recognition like activity profiling and DNA and data utilization for crime detection. Indeed of numerous publications in $\mathrm{NN}$ utilization in diverse medical challenges, however there is some reviewer available that demonstrates architecture in enhancing approaches like performance, accuracy, specificity and sensitivity. Henceforth, detection capability is normally referred as computing or sub-domain in computer vision to understand, locate, classify or targeted objects. Instance in detection tasks, image is scanned to identify some special characteristics or features.

\section{Biometrics}

Biometrics utilizes various techniques for unique human recognition based on more intrinsic behavioural or physical traits. Specifically in computer science, it is utilized as access control and identity access management. It is utilized to recognize individuals in groups under surveillance.

\section{J. Robotic Process Automation}

RPA utilizes scripts and other approaches to assist automate human action for effectual business processes. At present, it is utilized to execute task.

\section{K. Text Analytics and NLP}

NLP supports text analytics by offering understanding of sentence meaning and structure, sentiment and intent via ML and statistical approaches. At present, it uses fraud detection and security, an extensive range of automated assistants and applications for unstructured mining data.

\section{Education}

It provides grading, providing educators in many times. It access students and adapt its need in assisting them to work at own pace.

\section{Robotics}

AI facilitates resolves confronts in aging population and provides longer independence. It reduces traffic accidents and deaths, facilitates disaster response for dangerous situations. For instance, nuclear melts at fukushima power plant.

\section{Table I: Tabular representation of AI learning methods}

\begin{tabular}{|c|c|}
\hline S. No & AI learning methods \\
\hline 1 & Failure Driven Learning \\
\hline 2 & Learning by being Told \\
\hline 3 & Learning by Exploration \\
\hline
\end{tabular}

Table II: Tabular representation of Conventional methods and descriptions

\begin{tabular}{|c|c|c|}
\hline S. No & Methods & Descriptions \\
\hline 1 & Classical methods & Designing AI \\
\hline 2 & Connectionist methods & Developing AI \\
\hline
\end{tabular}

\section{Future of AI in Dentistry}

Based on previous report that is marked as era for AI evolution conducted with numerous investments. Cloud computing have offered organized access to huge data sets essential to train ISs. Data needs huge amounts of storage, turn out to be not cheap however faster in retrieval [12]. Healthcare is an extensive customer for AI applications.

With competency to examine huge amount of diagnostic images like CT scans, X-rays and MRIs, systems like this turned out to be probable field of concern, raising both probability of detection and speed. To motivate investigators of medical decision support, forecast that AI will rise tenfold.

AI based dentistry has platform for detection as final clinical evaluation. This development commences and investigators learned that educating computer in dentistry single dimension isn't so easy. However, there is an important progress toward appropriate for assistance in dental practice. 
Recently, man vs. detection challenge is presented pitted 3 dentists against Dentistry. Machine outperformed in "sensitivity," measures proportion of accurately predicted in contrast to samples in ground truth. They are successful in "precision" (ratio of appropriate predictions Vs predicted), however machine was comparable to dentists in precision. System maintains to improve and learn. Availability of detection tool sourced on AI in next eras. Detection of bone loss and periodontal disease attain are not behind.

It is now determined that psychological and psychosocial parameters may manipulate periodontal disease. For instance, patient with huge negative life events and patients with higher levels of stress are considerably more likely to possess periodontal disease. Fewer investigations have been associated these factors and outcome of periodontal treatment. Emotional is distinct as capability to control, perceive and estimates emotions. With this the term Emotional Intelligence (EI) comes into existence. EI is a subset of practical, social and personal intelligence, which helps by regulating and generating emotions to support intellectual and emotional growth. A person with higher EI has improved ability to manage, perceive and understand emotions; and less likely to deal with problematic characteristics, have verbal and other types of intelligence, and possess prosocial interactions. Henceforth, aim of further study is to find association between various EI and standardization response of chronic periodontitis.

EI is partitioned into diverse fields: emotion competencies, beliefs and values, and from this all fields are further divided into diverse domains. "emotional awareness" comprises of emotional awareness, assessment awareness of others as interpersonal management, domains \& intra-personal management.

It is known psychological stress regulate immune response. Communication amongst immune system and central nervous system happens through complexity of signals connecting endocrine, nervous \& immune systems. Disorder network homeostasis changes immune function.

Relationship among stress and periodontal disease has be proven, partially based on individuals lack with various periodontics features and complex to quantifying amount and stress duration. Moreover, various factors influences periodontal disease severity and exists uncertainty in individual's commencement. However, it is not probable to physical stress effects from EI in individuals.

Moreover, it is systemic diseases related disease like diabetes, $\mathrm{CV}$ disease and shares psychosocial stress as general risk factor in Fig 6.

In $\mathrm{AI}$, complete interpretation process is automated to calculate image to identify pathologies accurately and quickly. Obviously, stage is proliferation of applications. Use of AI-based technologies is common place for practice management.
DL tools with AI assist in treatment and diagnosing by facilitating detection of bone loss and modifications. Peri-implantitis is benefit in implant dentistry. In orthodontics, predictive models for tooth movement improve treatment planning. Using DL analysis, oral cancer is direct to detection and precise diagnosis of lifesaving implications.

\section{N. Fusion of AI and cloud}

$\mathrm{AI}$ and $\mathrm{CC}$ are considering two favourite components. Most of them imagine that cloud computing and AI businesses are completely unrelated. However, there is no industry where AI is not part of it. Starting from travel and tourism to healthcare, investigators now how AI is revolving all fields to each individual. Ascent of $\mathrm{CC}$ has illustrated critical factor in raising business sectors termed as 'cloud-local' as symbol of respect. For certain companies, ability to move infrastructure easily facilitated to move competitors, enormous amount of competitors battled in endeavour to cloud into difficult frameworks.

Cloud is essential for AI two ways: Initially, data sets are utilized in event was not for cloud, and also, cloud empowers organizations to adapt scalability needed data services to customers at expenses. Noticeably, one element maintains AI from reaching mass inside companies with aptitudes. This shows organizations know how to utilize AI and don't have application construction or algorithms to generate outcomes.

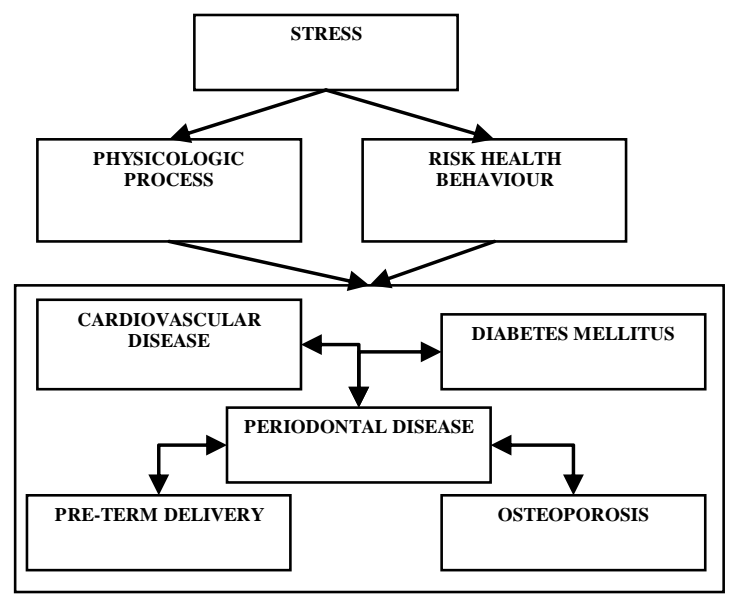

Fig 6: Disease related to periodontal diseases

Some start ups companies have established this AI and Cloud to flourish. For instance, Veritone has operating system with cloud-based CC system which utilizes enormous datasets from diverse sources. Veritone beliefs maximum capacity of cloud is open when it is accessible to foundations, organizations and people when information is not understood by machines.

Cloud and AI blend absolutely in numerous ways and in accordance to experts; AI might be a technology to revolutionize cloud computing solutions. AI as service enhances prevailing cloud computing solutions and provokes novel paths to development. 


\section{Conclusion}

Based on above discussions, ANN application fields measured in this study includes; medical science, emotional intelligence, computer security, finance, business, management, dentistry, cloud, facial alignment and so on. It is appealing to identify that $\mathrm{NN}$ data analysis enhances processing speed, accuracy, latency, tolerance, performance, volume and scalability. Numerous new and improved data management and analysis methods assist in ANN.

Henceforth, sourced on data analysis factors such as speed, accuracy, performance, latency, tolerance, volume and scalability, an assessment was carried out over ANN techniques. Other areas of further investigation by various investigators include:

(i) GA for superior performance concerning efficiency and efficiency.

(ii) Brain research concentrating on regression learning and adaptive dynamic programming and RL to offer effectual success in carrying out optimization.

(iii) HNNs models for superior performance in terms of competence and effectiveness.

(iv) Possible interest of research in integration of NNs with developing technologies.

(v)There is necessity for future research into ANN applications in businesses, industries, energy and wealth creation and agriculture.

(vi) Research concentrates on demonstrating if more beneficial trading system can be executed with expert systems, hybrid intelligent systems, merging FL and ANNs and genetic algorithms.

(vii) Research to investigate opportunity of utilizing ANNs to predict expert systems integrations and FL in demonstrating signals.

(viii) Possibility of utilizing GAs to choose optimal parameters and input variables for system.

In future, AI play fundamental role in creation and in software fields. AI will offer opportunities for AI technology and global technological parity can become future in all environment, public safety health domains and security.

\section{REFERENCES}

1. Hany H Ammar, Walid Abdelmoez and Mohamed Salah Hamdi, "Software Engineering Using Artificial Intelligence Techniques: Current State and Open Problems" - ICCIT 2012.

2. Y. LeCun, Y. Bengio, and G. Hinton, "Deep learning," Nature, vol. 521, no. 7553, pp. 436-444, May 2015.

3. C. Szegedy et al., "Intriguing properties of neural networks," presented at the International Conference on Learning Representations, 2014, pp. 1-10.

4. K. Crawford, "Opinion I Artificial Intelligence's White Guy Problem," The New York Times, 25-Jun-2016.

5. B. M. Lake, T. D. Ullman, J. B. Tenenbaum, and S. 1. Gershman, "Building machines that learn and think like people," Behav. Brain Sci., vol. 40, ed 2017.

6. W. Samek, T. Wiegand, and K.-R. Muller, "Explainable Artificial Intelligence: Understanding, Visualizing and Interpreting Deep Learning
Models," ITU J. ICT Discov. - Spec. Issue I - Impact Artif. Intell. AI Commun. Netw. Serv., vol. I, pp. 1-10, Dec. 2017.

7. G. Montavon, W. Samek, and K.-R. Muller, "Methods for interpreting and understanding deep neural networks," Digit. Signal Process, vol. 73, pp. 1-15, Feb. 2018.

8. E. Strumbelj and I. Kononenko, "Explaining Prediction Models and Individual Predictions with Feature Contributions," Knowllnf Syst, vol. 41, no. 3, pp. 647-665, Dec. 2014.

9. M. D. Zeiler and R. Fergus, "Visualizing and Understanding Convolutional Networks," in Computer Vision - ECCV 2014, 2014, pp.818-833.

10. W. Guo, K. Zhang, L. Lin, S. Huang, and X. Xing, "Towards Interrogating Discriminative Machine Learning Models," ArXiv 170508564 Cs Stat, May 2017.

11. Point, T. (2017, July 23). “Artificial Intelligence Overview” Retrieved August 22, 2017.

\section{AUTHORS PROFILE}

K .G. Rani Roopha Devi Research Scholar, Madurai Kamaraj University, Madurai.

Dr.R.Mahendra Chozhan ${ }^{2}$ Chozhan Dental Clinic, Kodaikanal, Periyakulam, Lakshmipuram

Dr. R. Murugesan Associate Professor, Department of Computer Science, C.P.A College, Bodinayakanur, 